\title{
Fall Detection and Daily Living Activity Recognition using Machine Learning
}

\author{
Ranjeeth Kumar. C, SathyaPraba. D, Shanmugapriya.S, Sounderya. R
}

\begin{abstract}
Elder people are increasing all over the world as a result certain fall occur in their daily life. This fall lead to several severe problems. The fall may often causes injuries and in many cases it result in death of the individual. The problem should be addressed to reduce the fall. By using some Machine Learning(ML) algorithm the fall and daily living activities are recognized. The acceleration and angular velocity data obtained from the dataset are used to detect the fall and daily living activity. Body movement of the person are collected and stored in the dataset. Acceleration and angular velocity data are used to extract the time and frequency domain feature and provide them to classification algorithm. Here, Logistic regression algorithm is used for detecting the fall and living activity. It is very effective algorithm and does not require too many computational resources. It is easy to regularize and provide well calibrated predicted probabilities as output. The sensitivity, accuracy and specificity of fall detection and activity recognition is obtained as a result. The performance evaluation is made with three classification algorithm. The three classification algorithm are Artificial neural network (ANN), K-nearest neighbours (KNN), Quadratic support vector machine (QSVM). Logistic regression provides highest accuracy compared with other three algorithm.
\end{abstract}

Keywords: fall detection, activity recognition, logistic regression, dataset values.

\section{INTRODUCTION}

Fall and daily living activity are recognized using several techniques. In this context machine learning framework is used for detecting the fall and daily living activity recognition. Logistic regression algorithm will result output either as 0 or 1 . Here the dataset values are used and the activities like fall, walking, jogging, jumping, walking upstair, walking downstair, standing to sitting, sitting to standing are recognized. All the above activities are related to classes and each class is given a separate values and logistic regression classifier is applied to the classes to detect the daily living activity. The dataset contains values which is based on the movement of the body. Other techniques are placing sensor on the body of the person and detect the fall but this often lead to failure. Another technique is placing sensor on the human and detect the fall by integrating sensor with smartphone.

Revised Manuscript Received on August 15, 2020.

* Correspondence Author

Ranjeeth Kumar. C*, Assistant Professor (Sr. Gr), Department of Information Technology, Sri Ramakrishna Engineering College, Chennai, India.

Sathya Praba. D, Student, Department of Information Technology, Sri Ramakrishna Engineering College, Chennai, India.

Shanmugapriya.S, Student, Department of Information Technology, Sri Ramakrishna Engineering College, Chennai, India.

Sounderya. R, Student, Department of Information Technology, Sri Ramakrishna Engineering College, Chennai, India.

(C) The Authors. Published by Blue Eyes Intelligence Engineering and Sciences Publication (BEIESP). This is an open access article under the CC BY-NC-ND license (http://creativecommons.org/licenses/by-nc-nd/4.0/)
This may cause failure when there is problem in the smartphone. By using machine learning algorithm fall detection is simple and provide good accuracy when compared with other techniques.

Logistic regression relate the value of dependent and independent variable using estimated probabilities of logistic function. This logistic function is also called sigmoid function. The probability value is transformed to binary value. The binary value range between 0 and 1 . The values are converted to either 0 or 1 based on threshold value.

\section{MOTIVATION}

This section provides an introduction of the existing methods in fall detection and elaborates the challenges

\section{Context-Aware Techniques}

It is a second main category of fall detection system. This system is based on sensors placed in the area around the people and monitor the fall occur. The sensors used for monitoring is encompass-floor sensors, pressure sensors, microphones, and cameras. We can include single or many type of sensor which deployed in specific areas. This system will be impossible when the person leaves the area. The main purpose of the system is video surveillance. We have to monitor the fall through the camera, it is used to capture the series of images which is subsequently processed by classification algorithm.

\section{Smart-phone Techniques}

Erhan, Aydin and Ibrahim alpher [12] proposed method to detect the fall using smart phones. The most challenging technique is the Human -centered computing and it is used to understand the human behavior and their social context with computer systems. In this paper, we introduced available dataset for HAR using smart phone and acknowledged results using SVM (Support Vector Machine) approach. The multiclass SVM employed for the classification of smart phone inertial data showed a recognition performance similar to previous work that has used special purpose sensors. Moreover, the dynamic activities can be efficiently classified to the newly introduced features in the released dataset, non-dynamic actions still present misclassification overlaps. The results have indicates that the computational complexity for the application will be assessed in the upcoming system.

\section{Wearable Sensor Techniques}

OlukunleOjetola, Elena Gaura and James Brusey[13] concluded that wearable sensors are mostly used to detect the fall. Many attempts with insufficient success made the challenge to sufficient dataset. The description of dataset to be simulation of falls, near-falls and ADL (Activities of Daily Living).

\section{Published By:}

Blue Eyes Intelligence Engineering

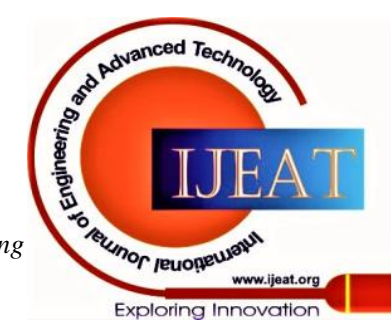


Four types of falls are left, right, forward, backward and ADL will be simulated. we will use this dataset in further work to develop machine learning algorithm. Experimental results have indicated that the proposed algorithms will give excellent results over agood set of activities and sensor type for monitoring the health.

\section{SisFall Technique}

Angela Sucerquia, Jose David Lopez and Jesus Francisco Vargas-Bonilla [14] concluded that the fall and movement dataset acquired with some people and the data is acquired with an accelerometer fixed into their body and along this data, a 5HZ filter keeps more information for detecting falls on elder people. The training fall detection algorithm with young people is not adequate on independent elder people. So finding maximum accuracy in fall detection algorithm is not a correct measure for real world application. The result indicates that the proposed system will give the good measure for real application

\section{PROPOSED SYSTEM}

Fall detection and daily living activity recognition is done by using one of the machine algorithm called logistic regression. The dataset values are used by the algorithm. The values are the body movement of the person, it the acceleration and angular velocity values. The dataset are trained using logistic regression and the trained dataset is tested to detect the fall. There are totally seven activities used in the algorithm. Each activity is given a class name and it is trained and tested. Two variable namely $\mathrm{X}$ and $\mathrm{Y}$ are used in the process of training and testing. This variable are called dependent and independent variable. Logistic regression uses dependent and independent variables and gives the result as binary values that lies between 0 and 1 . By using logistic function called sigmoid function either 0 or 1 will be the result of the function all these are done by using the estimated probability values.

\section{Logistic Regression}

Logistic regression model is a regression model to predict the probability that a given data entry belongs tothe category numbered as "1". Logistic regression model the data using sigmoid function. Logistic regression will become classification technique only when it attains a threshold level. Threshold level is fixing the value either as 0 or 1 . Usually logistic function result the value between 0 and 1 . The value will be fixed either as 0 or 1 based on sigmoid function also known as logistic function. The value is called threshold value.

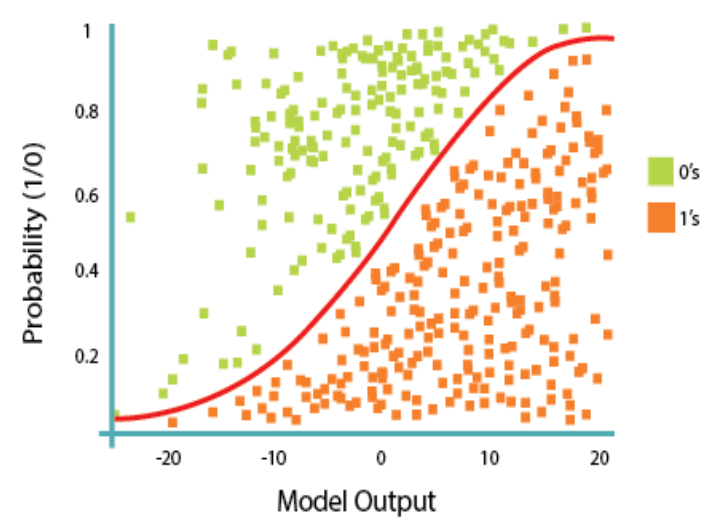

Fig1. Logistic regression
The above figure explains that the result of the logistic function will be 0's and 1's. Logistic regression can be classified as binomial, multinomial and ordinal. Threshold value is attained using precision value. There are two types of precision Low Precision/High Recall and High Precision/Low Recall. In applications where we want to reduce the number of false negatives without necessarily reducing the number false positives a decision value with low value of Precision or high value of Recall is choosed. In applications where we want to reduce the number of false positives without necessarily reducing the number false negatives, a decision value with high value of Precision or low value of Recall is choosed.

Features in the dataset are selected and fall detection is attained. The features are selected using bias intercept. Dataset contain body movement of the person and it will be in the form of acceleration and angular velocity. By using acceleration and angular velocity value falls are detected. Dataset is splitted into training and testing data and logistic regression is applied to the splitted dataset.

$$
\begin{gathered}
L\left(y_{i}, y_{i}^{*}\right)=\frac{-1}{n} \sum_{i=1}^{n} \log \left(1+e^{-y_{i}\left(w^{T} x_{i}+b\right)}\right) \\
y_{i}: \text { Label of point } i \in\{-1,+1\} \\
y_{i}^{*}: \text { Model prediction }=w^{T} x_{i}+b \\
w: \text { Weight vector } \\
x_{i}: \text { Input vector } \\
b: \text { Bias / Intercept }
\end{gathered}
$$

Fig2. Feature selection

Above figure explains the feature selection from the dataset and training the dataset using the vector value.

\section{Data Preprocessing}

Normalization is a common technique used in data preprocessing. Data isn't normally distributed, by using normalization technique the information is generally distributed. To scale the information min and max of every column is calculated and normalize each value of a column, min value is subtracted from each value and divided by max-min value.

Normalization $=$ value $-\min / \max -\min$

\section{Data Splitting}

The data is sometimes split into training data and test data. The training set contains a known output and therefore the model learns on this data so it is to be generalized to other data soon. The test dataset (or subset) is present in order to test our model's prediction on this subset.

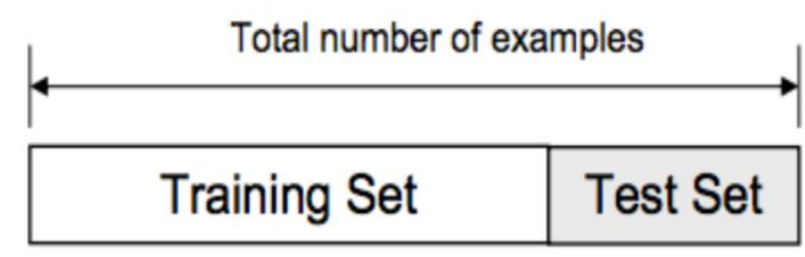

\section{Training the data}

Published By:
Blue Eyes Intelligence Engineering and Sciences Publication (C) Copyright: All rights reserved. 
The independent data set (X) is made. This is often dataset want to train the machine learning model(s).

To do this a variable called ' $\mathrm{X}$ ' is made, and convert the information into a numpy (np) array after dropping the 'Prediction' column, then store this new data into ' $\mathrm{X}$ '. Then the last 30 rows of knowledged data is faraway from ' $\mathrm{X}$ ', and store the new data back to ' $\mathrm{X}$ '. Last but not least the data has printed. The independent dataset is made within the previous step, now the dependent data set called ' $y$ ' are going to be created. This is often the target data, the one that holds longer term price predictions. To make this new data set ' $y$ ', the data will converted into a numpy array and from the 'Prediction' column, store it into new variable called ' $y$ ' and then remove the last 30 rows of knowledge from ' $y$ '. Then ' $y$ ' is going to be printed to make sure there are no NaN's. Now cleaned and processed data sets ' $X$ ' \& ' $y$ ' are generated. It is broke up into $80 \%$ training and $20 \%$ testing data for the model(s).

\section{Classification using Logistic Regression}

It is the ML (Machine Learning) algorithm and it is used for the classification problem. It's predictive analysis algorithm and it is supported to the concept of probability. Logistic regression is a classification algorithm may be a classification algorithm want to assign observations to a discrete set of classes. A number of Sample classificationRegression model but the Logistic Regression uses a more complex cost function, this cost function are often called as 'Sigmoid function' or also called as the 'logistic function' rather than the linear function. The hypothesis of logistic regression tends it to limit the value function between 0 and 1 . Hence linear functions fail to represent it as itcan have a worth greater than 1 or 0 which is not possible as per the hypothesis of logistic regression. The logistic function is additionally called as sigmoid function and it had been developed by statisticians to gauge the properties of increase in ecology, maxing out at the carrying capacity of the environment and rising quickly. The SF (Sigmoid Function) can take any real value and map into the value of range between 0 and 1 .

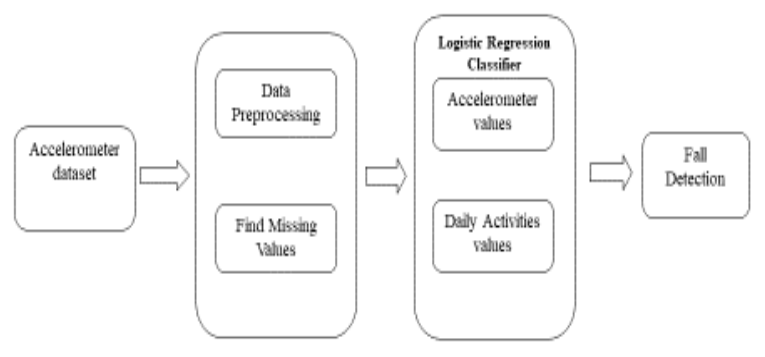

\section{Fig3. Block diagram for fall detection and daily living activity recognition}

\section{RESULTS ANDDISCUSSIONS}

This section shows the results and discussion of the proposed method.

\section{A. Experimental setup}

The proposed system is completed within the system with 2 GB RAM, Intel core i3 processor, Windows 7 or 8 Operating System, 120 GB hard disc. This technique is implemented using the software IDE-Anaconda and notebook.

\section{B. Data descript}

To detect the fall and daily living activity a dataset is required. Within the dataset acceleration and angular velocity data are present which represent the bowhich is employed to detect the fall when it occurs.

\section{Experimental results}

This section shows the sample results of the proposed method using testing dataset and training dataset. We had tested the performance through the activities like walking, falling, sitting, standing, walking upstairs, walking downstairs and lying are supported on acceleration and angular velocity. We have demonstrated the accuracy and precision of the classifier through graph.

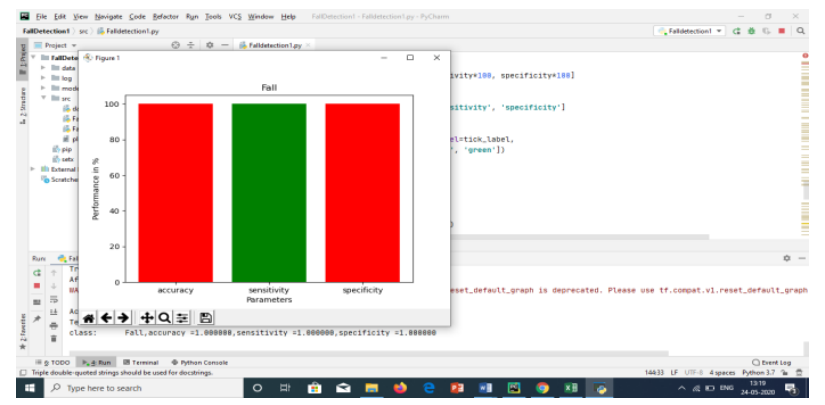

Fig4.Performance graph for Fall

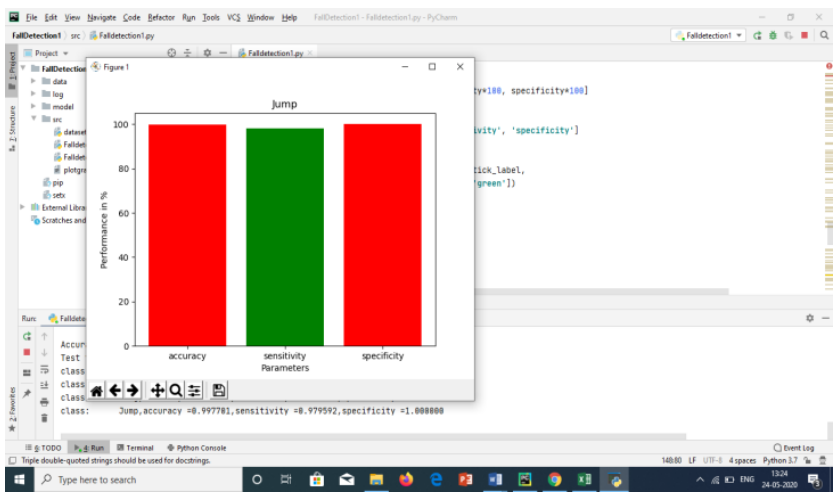

Fig5.Performance graph for Jump

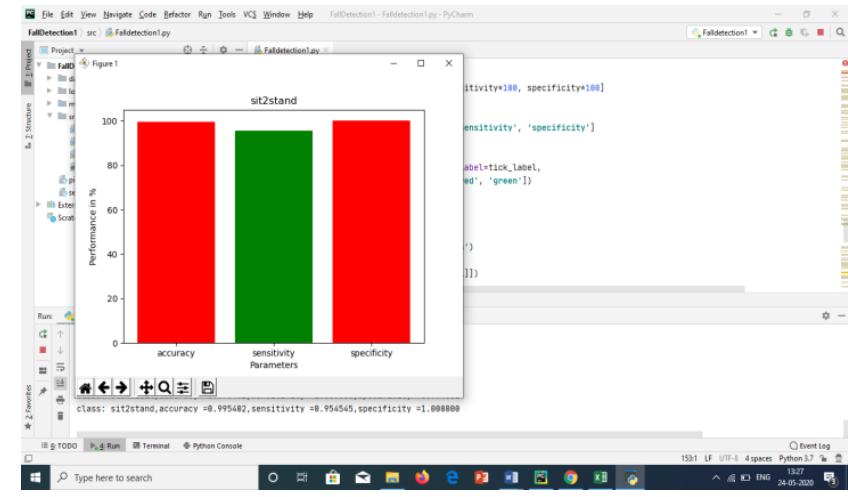

Fig6.Performance graph for sitting to standing

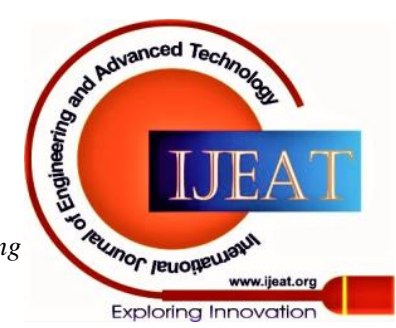




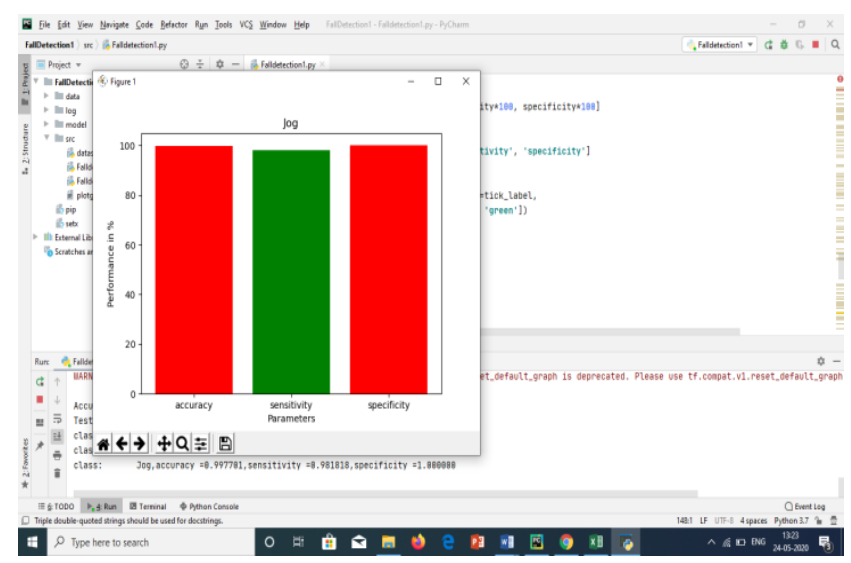

Fig7.Performance graph for jog

The above figures are the output of the logistic regression algorithm applied for the dataset. The results are displayed in the form of graph. Each graph represent the activities of the person. Totally seven activities can be recognized using this method. They are fall, jog, jump, walking upstair, walking downstair, sitting to standing and standing to sitting. Accuracy, sensitivity and specificity of the performed activity are also displayed in the graph. Accuracy is maximum for detecting fall and daily living activity recognition when logistic regression algorithm is applied.

\section{Dataset Description}

By using dataset values fall and activity recognition are performed. Dataset are splitted into training and testing dataset. This is the first and foremost step of this context. After splitting the data, dataset is trained by applying logistic regression classifier. The test dataset is executed and the result of test dataset is matched with trained dataset. By matching the training and testing dataset fall can be detected.

\section{Performance Results}

Accuracy: The measure of correctness of the detection is accuracy and it is given below

$$
\mathrm{TPR}=\frac{\mathrm{TP}+\mathrm{TN}}{\mathrm{TP}+\mathrm{FP}+\mathrm{FN}+\mathrm{TN}}
$$

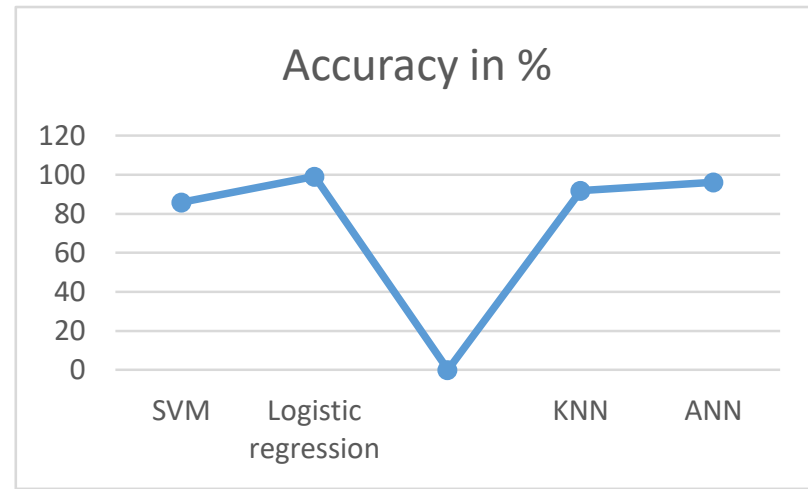

Fig8. Analysis of accuracy for the dataset based on the training percentage

TP represents true positive, $\mathrm{TN}$ is true negative, $\mathrm{FN}$ is false negative and FP is false positive

Sensitivity: The sensitivity of the TP rate (TPR) is defined as theNumber of positives identified correctly

$$
\mathrm{TPR}=\frac{\mathrm{TP}}{\mathrm{TP}+\mathrm{FN}}
$$

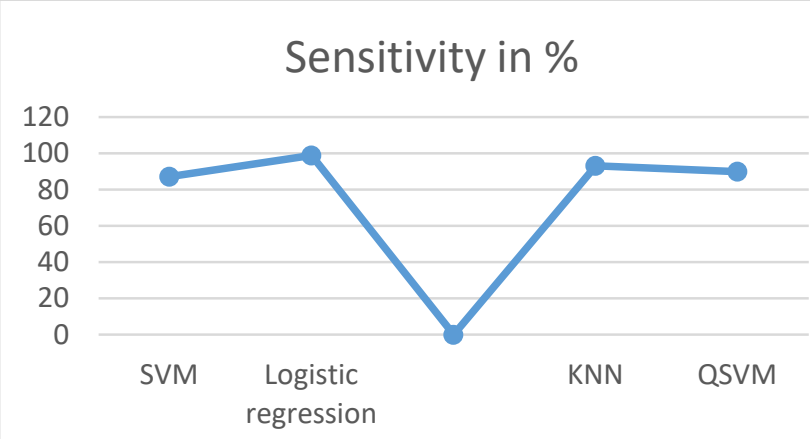

\section{Fig9. Analysis of sensitivity for the dataset based on the training percentage}

Specificity: the specificity or the TN rate (TNR) is defined as the number of number of negatives identified correctly

$$
\mathrm{TNR}=\frac{\mathrm{TN}}{\mathrm{TN}+\mathrm{FP}}
$$

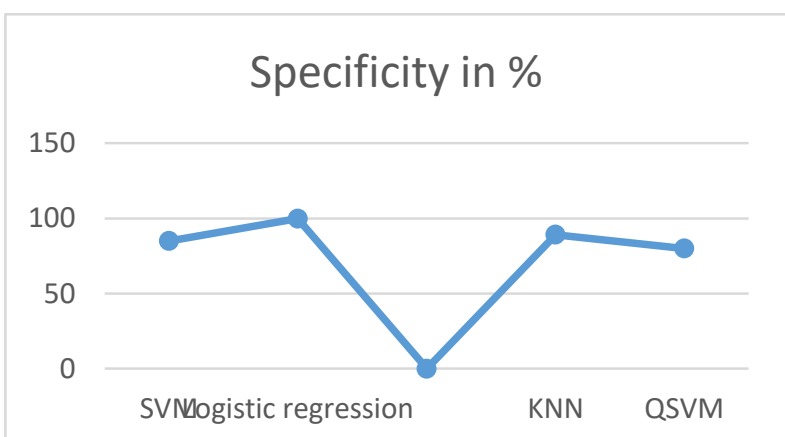

Fig10. Analysis of specificity for the dataset based on the training percentage

For the dataset, the proposed method has the maximum accuracy, sensitivity, specificity than the comparative methods.

\section{CONCLUSION}

Fall detection and daily living activity recognition is done using machine learning algorithm called logistic regression. This algorithm detects the value by training and testing the dataset values. The values are the body movement of the person that are stored as acceleration and angular velocity. Logistic regression algorithm is applied for the dataset and the result will be displayed in the form of graph. The resultant graph contain three parameter called accuracy, sensitivity and specificity. Two variable is used in the process of training and testing. These variables are called dependent and independent variable. Logistic function also called sigmoid function is applied and the value between the range 0 and 1 will be the result and it is finalized either 0 or 1 based on the threshold value. Performance of the fall detection and daily living activities model is also found. As a result, the performance is comparatively higher than the other machine learning algorithm when compared with logistic regression model.

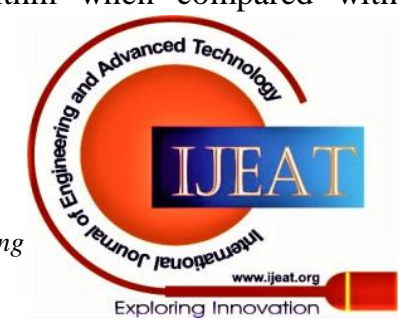


Three algorithms are compared with this model. These algorithms are artificial neural network (ANN), K-nearest neighbours (KNN), Quadratic support vector machine (QSVM). Therefore logistic regression gives highest accuracy and it is effective, highly interpretable, it doesn't require input features to be scaled,it doesn't require any tuning, it's easy to regularize, and it outputs well-calibrated predicted probabilities.

\section{REFERENCES}

1. Z. Luo, F. Branchaud-Charron, C. Lemaire, J. Konrad,S.Li,A.Mishra,A.Achkar,J.Eichel,and P.-M. Jodoin, "MIOTCD: A new benchmark datasetforvehicleclassificationandlocalization," IEEE Trans. Image Process., vol. 27, no. 10, pp. 5129-5141, Oct.2018.

2. R.Theagarajan,F.Pala,andB.Bhanu, "EDeN: Ensemble of deep networks for vehicle classification," in Proc. IEEE Conf Comput.Vis.Pattern Recognit. Workshops (CVPRW), 2017

3. P. L. St-Charles, G. A. Bilodeau, and R. Bergevin, "SuBSENSE: A univer- sal change detection method with local adaptive sensitivity,' IEEE Trans. Image Process., vol. 24, no. 1, pp. 359-373, Jan.2015.

4. Tom S.F. Haines and Tao Xiang, "Background Subtraction with Dirichlet Process Mixture Models", IEEE Transactions On Pattern Analysis And Machine Intelligence,Vol.36,No.4,April2014

5. Z. Dong and Y. Jia, "Vehicle typeclassification using distributions of structural and appearance-based features,' in Proc. IEEEInt.Conf. Image Process., Sep. 2013, pp. 4321-432

6. Y. Peng et al., "Vehicle type classification using data mining techniques," in The Era of InteractiveMedia.NewYork,NY,USA:Springer, 2013.

7. Florian Seidel Clemens Hage MartinKleinsteuber,"pROST: A Smoothed `p-norm Robust Online Subspace Tracking Method for Realtime Background Subtraction inVideo",March 29, 2013

8. D.Neelima, D. SanthiPriya "An ExtendedAndMultifeatures Background SubtractionWithSvm”,[Ijesat]InternationalJournalOfEng ineering Science \& Advanced Technology Volume- 2,Issue-5,1316 1326,IJESAT | Sep-Oct2012

9. Lucia Maddalena, Alfredo Petrosino, "The SOBS algorithm: what are the limits?" IEEE CVPR2012

10. Xinyi Cui, Junzhou Huang, Shaoting Zhang, and Dimitris N. Metaxas, "Background Subtraction UsingLowRankandGroupSparsityConstraints",

Fitzgibbonetal.(Eds.):ECCV2012,PartI,LNCS 7572, pp. 612-625, 2012,Springer-Verlag Berlin Heidelberg2012.

11. T. Bouwmans, F. El Baf, B. Vachon," Background Modeling using Mixture of Gaussians for Foreground Detection - A Survey", Laboratoire MIA, Université de La Rochelle, Avenue M.Crépeau, 17000 La Rochelle, France,12 Nov2008

12. Massimo Piccardi, "Background subtraction techniques: a review", The ARC Centre of Excellence for Autonomous Systems(CAS)Faculty of Engineering, UTS, April 15,2004

13. J. Yao and J.-M. Odobez, "Multi-layer background subtraction based on color and texture," in Proc. IEEE Conf. Comput. Vis. Pattern Recognit., Jun. 2007, pp. 1-8.

14. P. Wayne Power Johann, A. Schooners," Understanding Background Mixture Models for Foreground Segmentation", Proceedings Image and Vision Computing New Zealand2002, University of Auckland, Auckland, New Zealand 26-28th November 2002.S

15. Sebastian Brutzer, Benjamin H*oferlin, Gunther Heidemann Intelligent Systems Group, Universit"at Stuttgart, Germany,"Evaluation of BackgroundSubtraction Techniques for Video Surveillance",IEEE

\section{AUTHORS PROFILE}

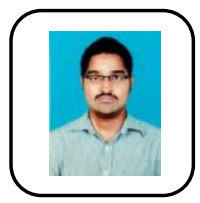

C. Ranjeeth Kumar is currently working as Assistant professor (Sr.Gr) in the department of Information Technology in Sri Ramakrishna Engineering College. He is currently pursuing Ph.D. degree in Anna University in the domain Artificial Intelligence. He obtained his Master of Engineering degree in Computer Science Engineering from Sri Ramakrishna Engineering college and completed his B Tech in Information Technology from Dr. Mahalingam college of Engineering and Technology, Pollachi. He has completed a consultancy project for International Aerospace Manufacturing Private Ltd. Bangalore. He has published papers in 8 International journals, presented papers in 3

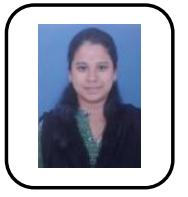

National Conferences. He is a life member of CSI, ISTE, IAENG and IACSIT. He has completed BEC with preliminary Certification and has NPTEL certifications.

D. Sathyapraba is pursuing her B Tech in Information Coimbatore, India. Technology from Sri Ramakrishna Engineering College in

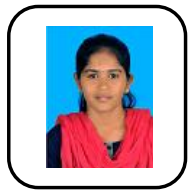

S. Shanmugapriya is pursuing her $B$ Tech in Information Technology from Sri Ramakrishna Engineering College in Coimbatore, India.

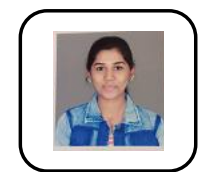

R. Sounderya is pursuing her B Tech in Information Technology from Sri Ramakrishna Engineering College in Coimbatore, India. 